\title{
Absence of Albumin Improves in Vitro Cellular Uptake and Disruption of Poloxamer 407-Based Nanoparticles inside Cancer Cells
}

\author{
Ana Loureiro, ${ }^{\dagger}$ Jennifer Noro, ${ }^{\dagger}$ Ana S. Abreu, ${ }^{\ddagger}$ Eugénia Nogueira, ${ }^{\dagger}$ Diana Soares da Costa, ${ }^{\S}, \| \odot$ \\ Carla Silva, ${ }^{\dagger}$ and Artur Cavaco-Paulo, ${ }^{*}+\odot$ \\ ${ }^{\dagger}$ Centre of Biological Engineering, University of Minho, Campus de Gualtar, 471 0-057, Braga, Portugal \\ ${ }^{\ddagger}$ Institute of Polymers and Composites (IPC) and Institute of Nanostructures, Nanomodelling and Nanofabrication (I3N), University \\ of Minho, Campus de Azurém, 4800-058 Guimarães, Portugal \\ §3B's Research Group-Biomaterials, Biodegradables and Biomimetics, University of Minho, Headquarters of the European Institute \\ of Excellence on Tissue Engineering and Regenerative Medicine, Ave Park, 4805-016 Taipas, Guimarães, Portugal \\ "ICVS/3B’s-PT Government Associate Laboratory, Braga/Guimarães, Portugal
}

Supporting Information

ABSTRACT: Novel nanoparticles based on Poloxamer 407 and vegetable oil were produced by high pressure homogenization. Functionalization of those nanoparticles was made by incorporation of folic acid (FA)-Poloxamer 407 conjugate. These nanoparticles showed suitable characteristics for intravenous therapeutic applications similarly to PEGylated albumin-based nanoparticles, previously described by our research group. Here, we found that the absence of albumin at the interface of Poloxamer 407-based nanoparticles improves the overall process of in vitro cellular uptake and nanoparticle disruption inside cancer cells (folate receptor, FR, positive cells). The results presented here suggest that interfacial composition of those nanoparticles is of paramount importance for drug trafficking inside cancer cells.

KEYWORDS: folic acid-Poloxamer 407 conjugate, folic acid-Poloxamer 407 nanoparticles, nanoparticle disruption, specific drug release, cancer therapy

\section{INTRODUCTION}

Polymer-based nanotechnology is growing as a promising area in pharmaceutical research. Block copolymers represent suitable agents for therapeutic applications, ${ }^{2,3}$ the Pluronic block copolymers (also known as "Poloxamers") being greatly proposed for controlled drug delivery. ${ }^{1,2}$ Poloxamers are widely used to facilitate gene/drug delivery, as emulsifying agents, or as agents helping in healing damaged cell membranes. Several advantages, such as nontoxic nature and high solubility in water, also increase the application of Poloxamers. ${ }^{4}$ Moreover, a pronounced sensitization to various anticancer agents was observed due to the interaction of these block copolymers with multidrug resistant (MDR) cells or tumors. ${ }^{5-7}$ Pluronic block copolymers disturb various drug resistance mechanisms such as microviscosity modification of the cellular membrane, abolishment of drug sequestration in cytoplasmic acidic vesicles, inhibition of drug efflux transporters, and inhibition of the glutathione/glutathione $S$-transferase detoxification system. ${ }^{5,8,9}$ Furthermore, these copolymers selectively affect MDR cells inducing a decrease in ATP levels, this reduction being one potential cause for chemosensitization of MDR cells. ${ }^{5,8}$

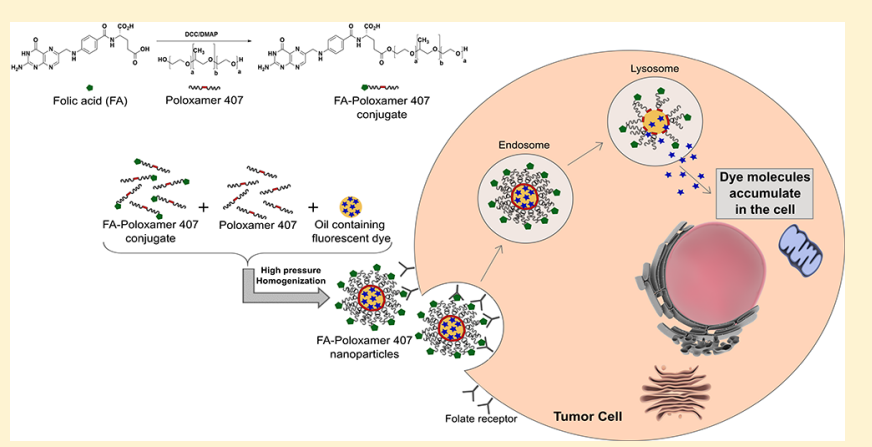

Poloxamers comprise hydrophilic poly(ethylene glycol) (PEG) and hydrophobic poly(propylene oxide) (PPO) blocks organized in a triblock PEG-PPO-PEG structure. The PEG and PPO chain lengths can vary, producing Poloxamers with different properties and functions. Their effect on cancer cells is affected by molar mass ratio between the PEG and PPO blocks and chemical compositions. ${ }^{9}$

Pluronic F127 (Poloxamer 407) has gained extensive consideration in biomedicine. ${ }^{10}$ The long PEG hydrophilic chains of Poloxamer 407 triblock copolymer improve its physical stability due to PEG repulsive property. ${ }^{6,11}$ Moreover, these long hydrophilic chains can enhance the blood circulation time in vivo of nanoparticles. ${ }^{11}$ Lipophilic drugs can be incorporated in the hydrophobic core, PPO part, of Poloxamer 407. These structural characteristics of Poloxamer 407 promote its application as a vehicle for drug delivery. Moreover, this

Received: October 11, 2017

Revised: December 18, 2017

Accepted: January 1, 2018

Published: January 1, 2018 
block copolymer can disturb various processes in the cells such as gene expression, mitochondrial respiration, activity of drug efflux transporters, and apoptotic signal transduction. ${ }^{12}$

In the present study, we report the role of interfacial albumin on nanoparticle composition. Poloxamer 407-based nanoparticles (prepared in the absence of albumin) were developed using a methodology previously described by us. ${ }^{13}$ The novel functionalized nanoparticles were produced by incorporation of a conjugate of Poloxamer 407 with folic acid (FA). Poloxamer 407-based nanoparticles were compared with PEGylated albumin-based nanoparticles, previously developed by our research group, by evaluation of in vitro cellular uptake and nanoparticle disruption.

\section{EXPERIMENTAL SECTION}

Conjugation of FA with Bovine Serum Albumin (BSA). The production of FA-BSA conjugate was performed as previously described. ${ }^{14}$ Dialysis (cutoff molecular weight 14 $\mathrm{kDa}$, against PBS for 8 days) was performed to remove the excess of FA and other reactants from the conjugate. Then, the solution was also purified using a gel filtration chromatography column (GE Healthcare, U.K.).

The quantitation of FA in conjugate was performed by measuring the absorbance at the maximum wavelength of FA $(350 \mathrm{~nm})$. The quantitation of BSA in conjugate was carried out using the Lowry method. ${ }^{15}$ The total protein concentration is shown by a color change of the sample solution in proportion to protein concentration, which can then be quantified by measuring the absorbance at $750 \mathrm{~nm}$, using a Synergy $\mathrm{Mx}$ Multi-Mode Reader (BioTek, USA).

Conjugation of FA with Poloxamer 407. The conjugation of FA with Poloxamer 407 (Sigma-Aldrich, USA) was performed between the hydroxyl group of Poloxamer 407 and the carboxyl group of FA, via an esterification reaction. In brief, FA (0.4589 g), $N, N^{\prime}$-dicyclohexylcarbodiimide (DCC, SigmaAldrich, USA) (0.2159 g), and 4-(dimethylamino)pyridine (DMAP, Sigma-Aldrich, USA) (0.131 g) were dissolved together in $20 \mathrm{~mL}$ of anhydrous dimethyl sulfoxide (DMSO, Sigma-Aldrich, USA). The solution was stirred at $30{ }^{\circ} \mathrm{C}$ in the dark for $2 \mathrm{~h}$ to activate the carboxylic groups of FA. After addition of $1.46 \mathrm{~g}$ of Poloxamer 407, the solution was stirred at $30{ }^{\circ} \mathrm{C}$ overnight. The precipitates in the solution were filtered. The solution was dialyzed (cutoff molecular weight $2 \mathrm{kDa}$ ) against PBS to remove the unreacted FA. After dialysis, the precipitates in the solution were removed by centrifugation at $6000 \mathrm{rpm}$ for $30 \mathrm{~min}$. Then, the solution was purified through a gel filtration chromatography column (GE Healthcare, U.K.).

The quantitation of FA conjugated with Poloxamer 407 was performed by measuring the absorbance at the maximum wavelength of FA (350 nm) using a Synergy Mx Multi-Mode Reader (BioTek, USA). The conjugate was also characterized by proton nuclear magnetic resonance $\left({ }^{1} \mathrm{H} \mathrm{NMR}\right)$, Fourier transform infrared spectroscopy (FTIR), and matrix-assisted laser desorption/ionization with time-of-flight (MALDI-TOF).

${ }^{1} \mathrm{H}$ NMR Analysis. Poloxamer 407, FA, and FA-Poloxamer 407 conjugate were characterized by ${ }^{1} \mathrm{H}$ NMR spectroscopy, using a Bruker Avance III $400(400 \mathrm{MHz})$. DMSO- $d_{6}$ was used as deuterated solvent, using the peak solvent as internal reference.

FTIR Analysis. FTIR spectra were obtained with a Jasco FT/IR-4100 spectrophotometer. Poloxamer 407, FA, and FAPoloxamer 407 conjugate (previously lyophilized) were mixed with potassium bromide $(\mathrm{KBr})$, which was used as matrix.
Before collection, background scanning was performed using $\mathrm{KBr}$ powder, and then $\mathrm{KBr}$ pellets were analyzed. The spectra were obtained in the region of $400-4500 \mathrm{~cm}^{-1}$ with a resolution of $4 \mathrm{~cm}^{-1}$ at room temperature.

MALDI-TOF Analysis. Mass/charge of Poloxamer 407, FA, and FA-Poloxamer 407 conjugate was verified by MALDITOF using super-2,5-dihydroxybenzoic acid (super-DHB) as matrix $(\geq 99.5 \%)$. The mass spectra were acquired on an Ultraflex MALDI-TOF mass spectrophotometer (Bruker Autoflex Speed, Daltonics GmbH, Germany) equipped with a $337 \mathrm{~nm}$ nitrogen laser. Each sample, previously dissolved in $30 \%$ acetonitrile $/ 70 \%$ trifluoroacetic acid (TA30), was mixed (1:1) with a saturated solution of super-DHB in TA30. Each mixture $(2 \mu \mathrm{L})$ was spotted onto the ground steel target plate (Bruker part no. 209519) and analyzed using the linear positive mode.

Nanoparticle Preparation. A high-pressure homogenizer (APV-2000, Denmark) was used for the nanoparticle preparation. BSA and Poloxamer 407 were dissolved in PBS (pH 7.4) at concentrations of 10 and $5 \mathrm{mg} / \mathrm{mL}$, respectively. The aqueous solution was mixed with the vegetable oil and subjected to high pressure homogenization. Poloxamer 407based nanoparticles were produced by the emulsification of an aqueous solution of Poloxamer 407 at $5 \mathrm{mg} / \mathrm{mL}$ with vegetable oil.

FA-BSA conjugate solution, previously prepared, was added at $1: 100$ ratio $(\mathrm{m} / \mathrm{m})$ to the aqueous phase to obtain $F A$-tagged albumin-based nanoparticles. FA-Poloxamer 407 conjugate solution was also added to the Poloxamer 407 solution equaling the same initial concentration of FA used for albumin-based nanoparticle production.

Nanoparticles containing a fluorescent dye were produced by the introduction of the cationic dye Hoechst 34580 (SigmaAldrich, USA) or $1,1^{\prime}$-dioctadecyl-3,3,3',3'-tetramethylindocarbocyanine perchlorate (DiI, Sigma-Aldrich, USA) in the oil phase. The free dye was separated from the nanoparticles by passage through a gel filtration chromatography column (GE Healthcare, U.K.). A Synergy Mx Multi-Mode Reader (BioTek, USA) was used to perform the quantification of the free dye.

Physicochemical Characterization. Size distribution and zeta-potential of nanoparticles were analyzed as previously reported. ${ }^{14}$ Stability studies of these physicochemical characteristics were performed as before described. ${ }^{13}$

Nanoparticles were also characterized by nanoparticle tracking analysis (NTA), using a Malvern NanoSight NS500 instrument (Salisbury, U.K.), measuring both particle size distribution and concentration. The measurements were performed at room temperature, and the samples were $10000 \times$ diluted with water.

Cells and Culture Conditions. Human skin fibroblasts (BJ5ta cell line) (ATCC, CRL-4001) and a subclone of HeLa cells (KB cell line) (ATCC, CCL-17) were obtained from American Type Culture Collection (LGC Standards, U.K.). The cultures were maintained as previously described. ${ }^{13}$

Cell Viability Assay. BJ5ta cell viability was analyzed using the Promega CellTiter 96 AQueous Non-Radioactive Cell Proliferation (MTS) assay (Promega, USA) as previously reported. $^{13}$

Confocal Microscopy. The cellular internalization and disruption of nanoparticles were analyzed using nanoparticles loaded with DiI and Hoechst 34580, respectively. The KB cancer cell line, FR positive cells, was used in this study. The seeding of the cells was performed in 24-well tissue culture 
Scheme 1. (A) Scheme of the Proposed Mechanism of FA-Poloxamer 407 Conjugate Synthesis and (B) Schematic Illustration of the Preparation of FA-Poloxamer 407 Nanoparticles
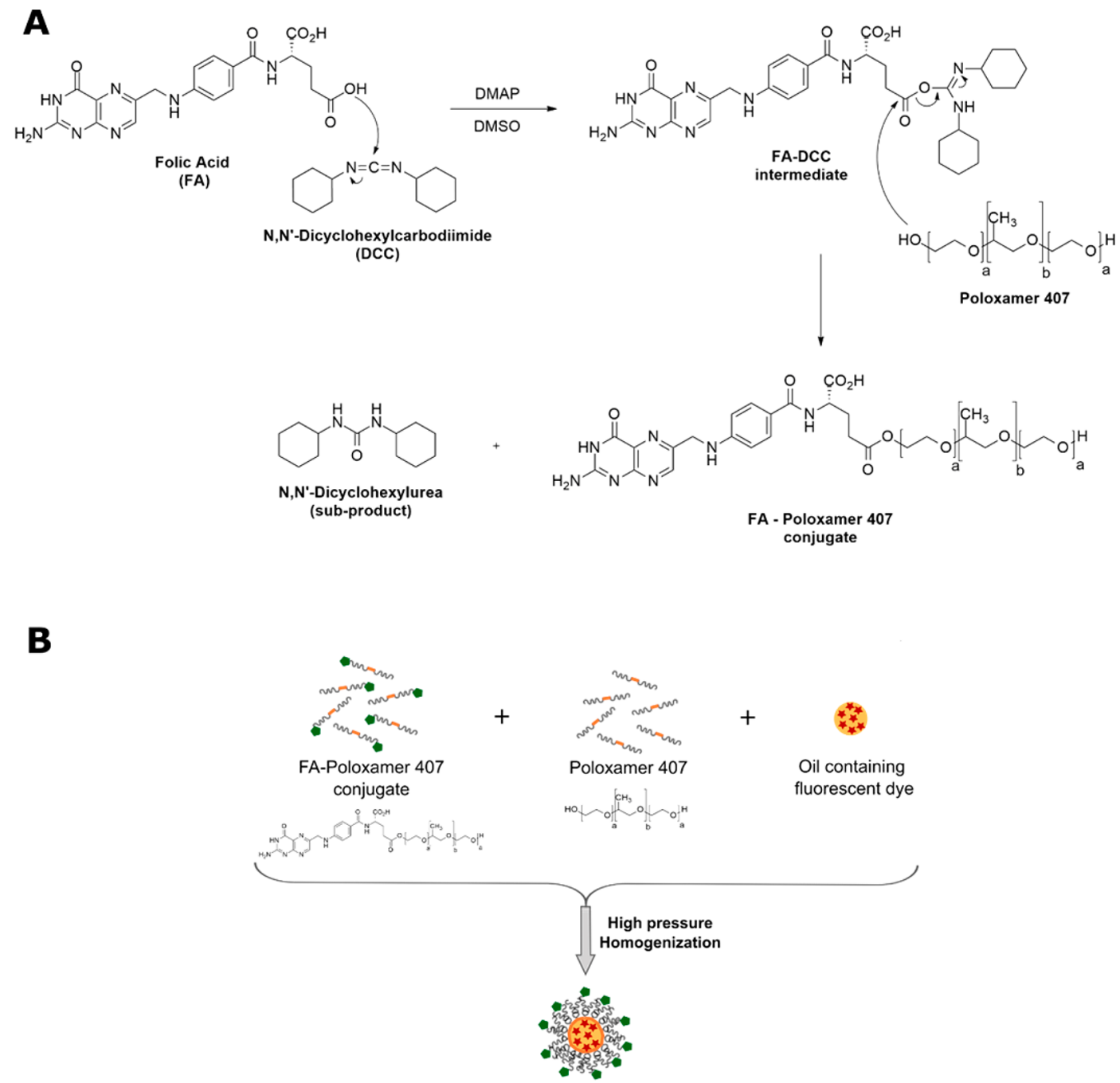

FA-Poloxamer 407 nanoparticle

polystyrene plates (TPP, Switzerland) at a density of $5 \times 10^{4}$ cells/well. Nanoparticles containing or not FA at the surface were diluted in FA-free Hanks balanced salt solution (HBSS medium) (Lonza, Belgium). After wash the cells with PBS buffer, $150 \mu \mathrm{g} / \mathrm{mL}$ of nanoparticles (based on Poloxamer 407 concentration) was added to the monolayer and incubated for 2 $\mathrm{h}$ at $37{ }^{\circ} \mathrm{C}$. Cells were washed two times with acid ( $\left.\mathrm{pH} 3.5\right)$ and neutral ( $\mathrm{pH} 7.4)$ PBS to remove nanoparticles that were not internalized. Cells were fixed with $4 \%(\mathrm{v} / \mathrm{v})$ paraformaldehyde, for $30 \mathrm{~min}$, and washed with PBS, and the coverslips were placed on slides coated with Permafluor mounting medium (Thermo Scientific, U.K.). For internalization evaluation of nanoparticles containing DiI, the Permafluor contained $5 \mu \mathrm{g} /$ $\mathrm{mL}$ of Hoechst. Samples were analyzed using an inverted Zeiss confocal laser scanning microscope (Olympus Fluoview FV1000).

Fluorescence Analysis. 2D maximum projections were used for the fluorescence analysis. Using ImageJ (v1.50e, NIH), each cell was selected and the area, the mean fluorescence, and the integrated fluorescence were measured, along with several adjacent background readings. The corrected total cellular fluorescence $(\mathrm{CTCF})=$ integrated density - (area of selected cell $\times$ mean fluorescence of background readings), was calculated for the different conditions. At least 25 cells were considered for each experimental condition. Statistical analysis (Shapiro-Wilk test for normality followed by Kruskal-Wallis test, $p<0.05$ ) was performed using GraphPad Prism 5.

\section{RESULTS AND DISCUSSION}

Synthesis and Characterization of FA-Poloxamer 407 Conjugate. The development of specialized nanoparticles for the treatment of cancer has been greatly exploited. The presence of a targeting agent in a nanoparticle surface can induce their accumulation in the tumor, in the tumor-bearing organ, in the individual cancer cells, in specific molecules of cancer cells, or in intracellular organelles. ${ }^{16} \mathrm{FA}$ is a great targeting agent for cancer tissues due to its high binding affinity to FRs at cell surfaces, which are overexpressed in several types of human cancers and have limited expression on normal tissues. $^{16,17}$

Poloxamer 407 has been greatly evaluated for diverse biomedical applications mainly due to its terminal hydroxyl groups. The chemical and/or covalent modification of these groups is often performed to attain active functionalities for targeted applications and improve the physical stability. ${ }^{12,18,19}$ Thus, in order to produce Poloxamer 407-based nanoparticles 


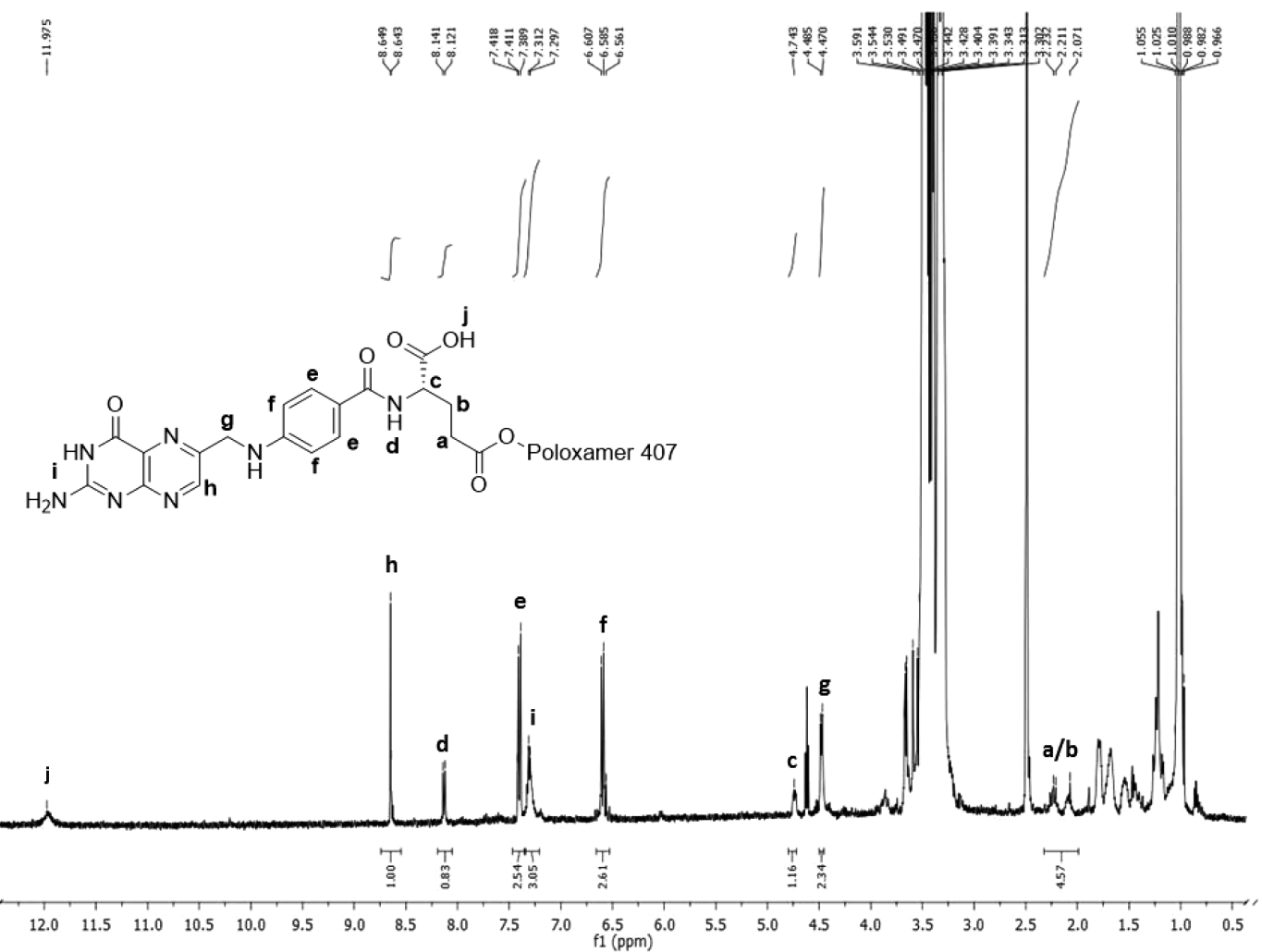

Figure 1. ${ }^{1} \mathrm{H}$ NMR spectrum of FA-Poloxamer 407 conjugate in DMSO- $d_{6}$. The peaks labeled in bold lowercase letters correspond to the protons indicated in the structure of FA.

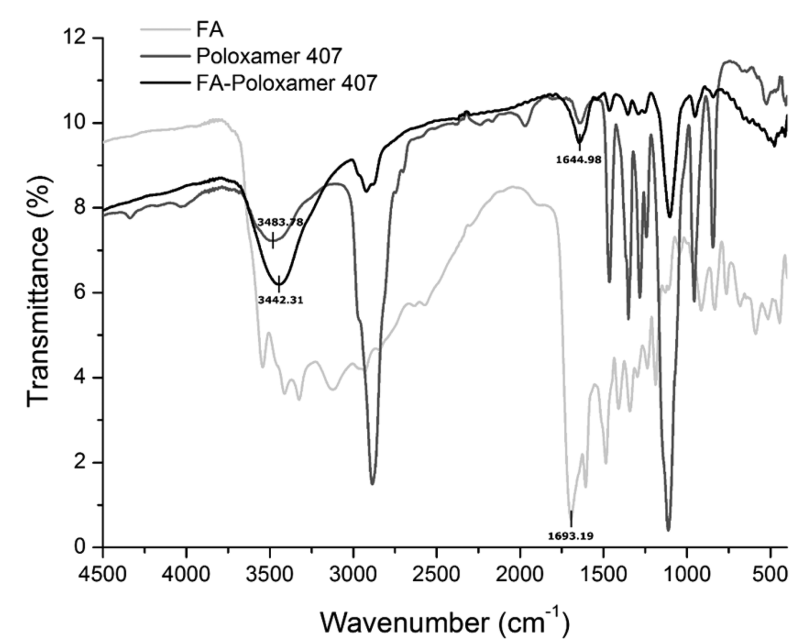

Figure 2. FTIR spectra of FA, Poloxamer 407, and FA-Poloxamer 407 conjugate.

that can be used for cancer therapy, we produced a FAPoloxamer 407 conjugate to incorporate into an initial formulation for nanoparticle production.

In Scheme $1 \mathrm{~A}$ is represented a proposal scheme of the mechanism of conjugation between FA and Poloxamer 407.

FA was conjugated to Poloxamer 407 via an esterification reaction between the carboxyl group of FA and the hydroxyl group of Poloxamer 407 using DMAP as the activator and DCC as the coupling agent. Considering the described accessibility of the $\gamma$-activated carboxyl group of $\mathrm{FA}^{20,21}$ we propose that the esterification reaction occurred via the $\gamma$ carboxyl group of FA when the $\gamma$-activated derivate was produced. An intermediate compound was produced, which reacted with Poloxamer 407. The products resulting from this reaction were the FA-Poloxamer 407 conjugate and urea, as subproduct.

After synthesis, the purified FA-Poloxamer 407 conjugate was characterized by ${ }^{1} \mathrm{H}$ NMR spectroscopy. Comparing the spectrum obtained (Figure 1) with the spectrum of FA, the main differences are related to the disappearance of one of the carboxylic group peaks $\left(\delta_{\mathrm{H}} \approx 12 \mathrm{ppm}\right)$. In the spectrum of the conjugate product, the only peak corresponding to this group is assigned at $\delta_{\mathrm{H}} 11.98 \mathrm{ppm}(\mathbf{j})$. Regarding the aliphatic protons, a and $\mathbf{b}$, both showed up in a very similar place, only with a slight deviation to a higher value. Proton $\mathbf{c}$ is assigned at higher chemical shift $\left(\delta_{\mathrm{H}} 4.74 \mathrm{ppm}\right)$ comparing to the starting material $\left(\delta_{\mathrm{H}} 4.38 \mathrm{ppm}\right)$. Concerning the aromatic protons of the phenyl group, e protons showed up at $\delta_{\mathrm{H}} 7.41 \mathrm{ppm}$ and $\mathbf{f}$ protons at $\delta_{\mathrm{H}}$ $6.60 \mathrm{ppm}$. In FA, these protons can be observed at $\delta_{\mathrm{H}} 7.64$ and $6.63 \mathrm{ppm}$, respectively. Aromatic amines (i) of the fused aromatic ring showed up at $\delta_{\mathrm{H}} 7.31 \mathrm{ppm}$ instead of $\delta_{\mathrm{H}} 6.92$ ppm. All other protons have the same chemical shift as in the original FA. Poloxamer 407 signals are in exactly the same place, indicating a large amount of unreacted Poloxamer 407. In stacked spectra (in Figure S1) it is possible to observe the differences among the reactants and the conjugate product. These results indicated that FA-Poloxamer 407 conjugate was achieved via the esterification reaction described.

The synthesis of FA-Poloxamer 407 conjugate was also confirmed by FTIR and MALDI-TOF analysis. Figure 2 shows the FTIR spectra of FA, Poloxamer 407, and FA-Poloxamer 407 conjugate. The spectrum of FA-Poloxamer 407 presents the characteristic absorption peaks of FA and Poloxamer 407. A deviation of the $\mathrm{C}=\mathrm{O}$ stretching vibration absorption of FA $(\nu$ $=1693.19 \mathrm{~cm}^{-1}$ ) was observed in the spectrum of FA- 


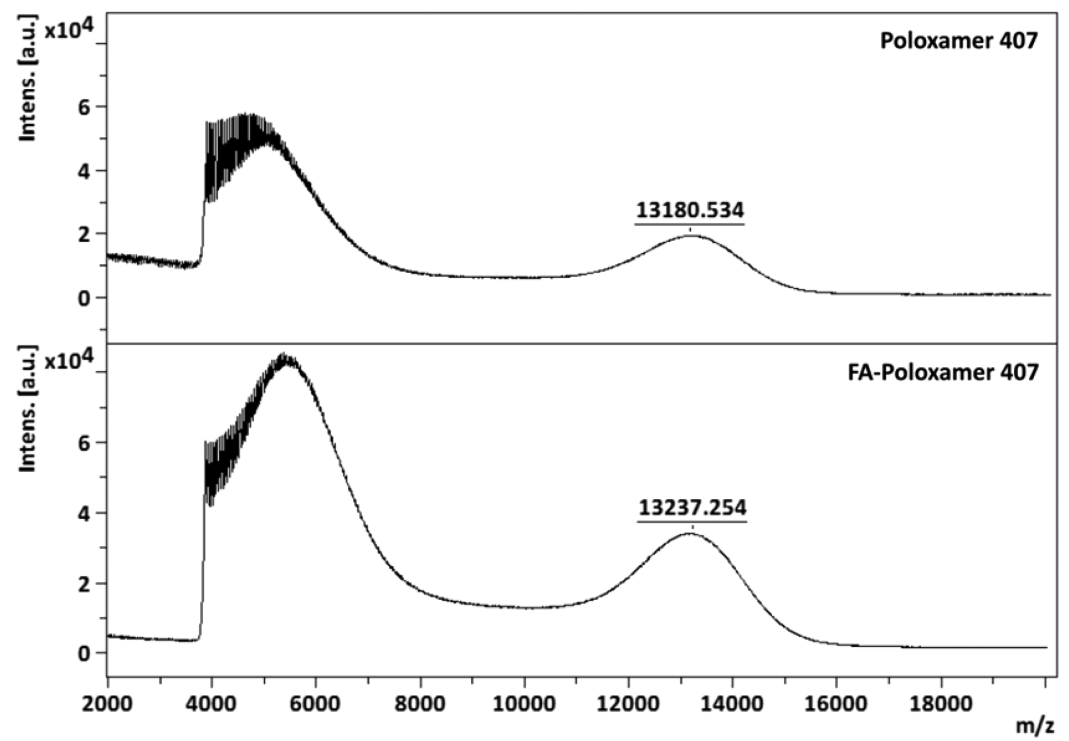

Figure 3. MALDI-TOF mass spectra of Poloxamer 407 and FA-Poloxamer 407 conjugate, acquired in linear positive mode.

Table 1. Physicochemical Characterization of Nanoparticles Evaluated by DLS and NTA Measurements ${ }^{a}$

\begin{tabular}{|c|c|c|c|c|c|c|}
\hline & \multicolumn{3}{|c|}{ DLS } & \multicolumn{3}{|c|}{ NTA } \\
\hline & $\begin{array}{c}\text { Z-average } \\
(\text { d.nm })\end{array}$ & $\begin{array}{l}\text { polydispersity índex } \\
\text { (PDI) }\end{array}$ & $\begin{array}{l}\text { zeta-potential } \\
(\mathrm{mV})\end{array}$ & mean $(\mathrm{nm})$ & std $\operatorname{dev}(\mathrm{nm})$ & $\begin{array}{c}\text { concn (E12 particles/ } \\
\text { mL) }\end{array}$ \\
\hline PEGylated albumin nanoparticles & $87.59( \pm 0.84)$ & $0.149( \pm 0.008)$ & $-1.23( \pm 0.73)$ & $81.67( \pm 2.08)$ & $28.67( \pm 0.58)$ & $6.47( \pm 0.24)$ \\
\hline $\begin{array}{l}\text { FA-PEGylated albumin } \\
\text { nanoparticles }\end{array}$ & $76.35( \pm 2.32)$ & $0.167( \pm 0.003)$ & $-1.45( \pm 0.27)$ & $85.00( \pm 2.65)$ & $31.67( \pm 1.53)$ & $6.45( \pm 0.27)$ \\
\hline Poloxamer 407 nanoparticles & $82.40( \pm 0.62)$ & $0.108( \pm 0.007)$ & $-1.30( \pm 0.19)$ & $84.00( \pm 4.24)$ & $32.25( \pm 2.06)$ & $6.15( \pm 0.39)$ \\
\hline FA-Poloxamer 407 nanoparticles & $77.18( \pm 1.27)$ & $0.131( \pm 0.003)$ & $-0.33( \pm 0.28)$ & $81.67( \pm 1.15)$ & $28.33( \pm 2.31)$ & $6.39( \pm 0.31)$ \\
\hline
\end{tabular}

${ }^{a}$ Comparison with previously reported PEGylated albumin nanoparticles. Values represent the mean \pm SD of three independent experiments.

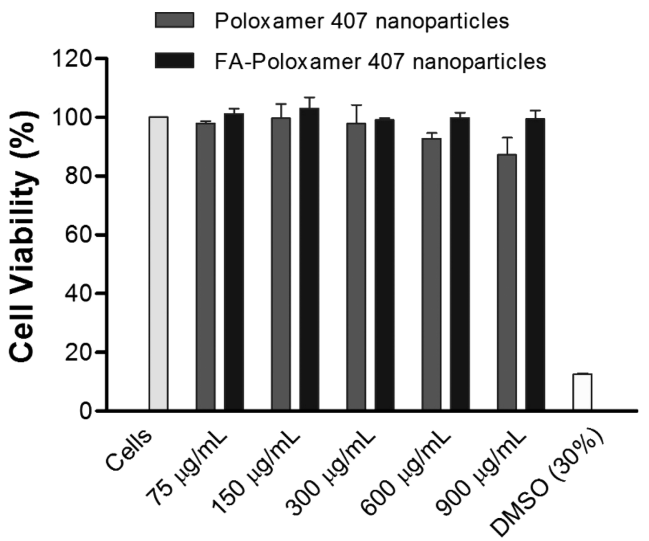

Figure 4. BJ5ta cell line viability after $72 \mathrm{~h}$ of contact with different concentrations of Poloxamer 407-based nanoparticles prepared with and without FA-Poloxamer 407 conjugate, compared with cells (negative control) and cells incubated with $30 \%$ (v/v) of DMSO (positive control), determined by MTS assay. Values are the mean \pm $\mathrm{SD}$ of two independent experiments.

Poloxamer 407 conjugate $\left(\nu=1644.98 \mathrm{~cm}^{-1}\right)$, confirming the formation of the FA-Poloxamer 407 conjugate. Moreover, a deviation of the $\mathrm{OH}$ stretching vibration absorption of Poloxamer 407 from $\nu=3483.78 \mathrm{~cm}^{-1}$ to $\nu=3442.31 \mathrm{~cm}^{-1}$ in the spectrum of the conjugate was also observed. These results evidence the occurrence of an ester linkage between FA and Poloxamer 407.
By analysis of MALDI-TOF spectra one could infer that $11.65 \%$ of the Poloxamer 407 molecules were modified with a molecule of FA (Figure 3). Apparently, just one molecule of FA was conjugated with each molecule of Poloxamer 407 since the mass difference between Poloxamer 407 and FA-Poloxamer 407 was very low. The mass difference obtained was lower than the molecular weight of FA because the sample was analyzed using the linear positive mode. In this detection mode, the ions of identical mass arrive at the detector at slightly different time points, causing peak broadening and limiting the resolution of the mass spectrum. From this spectrum we can only infer the value of medium molecular mass.

Preparation and Characterization of Poloxamer 407Based Nanoparticles. Poloxamer 407-based nanoparticles were produced by high pressure homogenization of Poloxamer 407 aqueous solution with vegetable oil, using the optimized conditions previously described by Loureiro et al. ${ }^{13}$ for albumin-based nanoparticle production. The characterization of these nanoparticles using DLS (Table 1) evidenced that Poloxamer 407-based nanoparticles and PEGylated albuminbased nanoparticles presented similar physicochemical characteristics. Nanoparticles showed narrow and small size, neutral surface charge (zeta-potential values close to zero), and high stability during storage ( $>20$ weeks), making them suitable for intravenous application.

For the production of nanoparticles containing FA as targeting agent, purified FA-BSA and FA-Poloxamer 407 conjugates were added to BSA solution containing Poloxamer 407 and Poloxamer 407 solution, respectively. The FA-BSA 
A
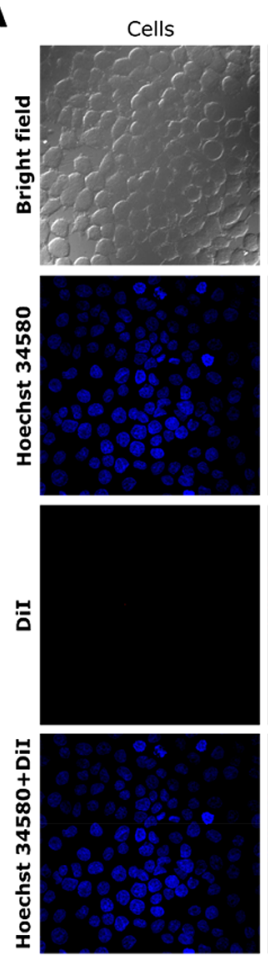

PEGylated albumin FA-PEGylated albumin nanoparticles
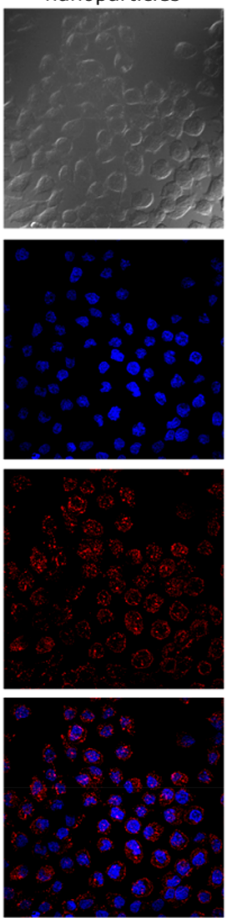

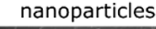

nanoparticles
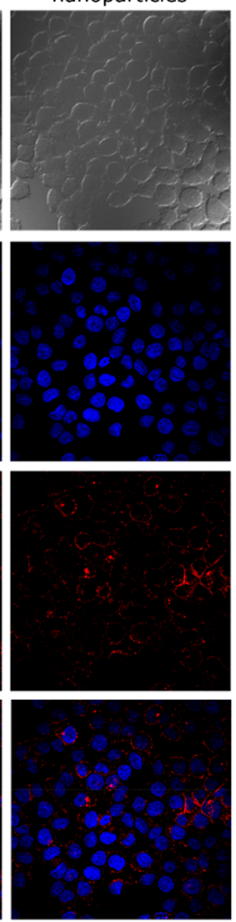

Poloxamer 407

nanoparticles
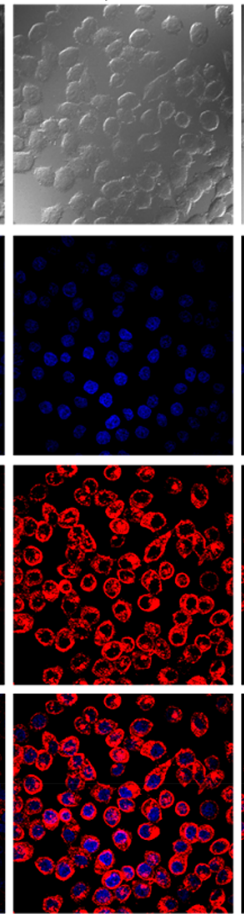

FA-Poloxamer 407

nanoparticles
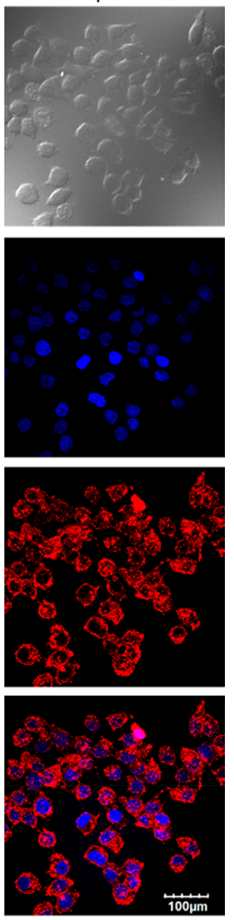

B

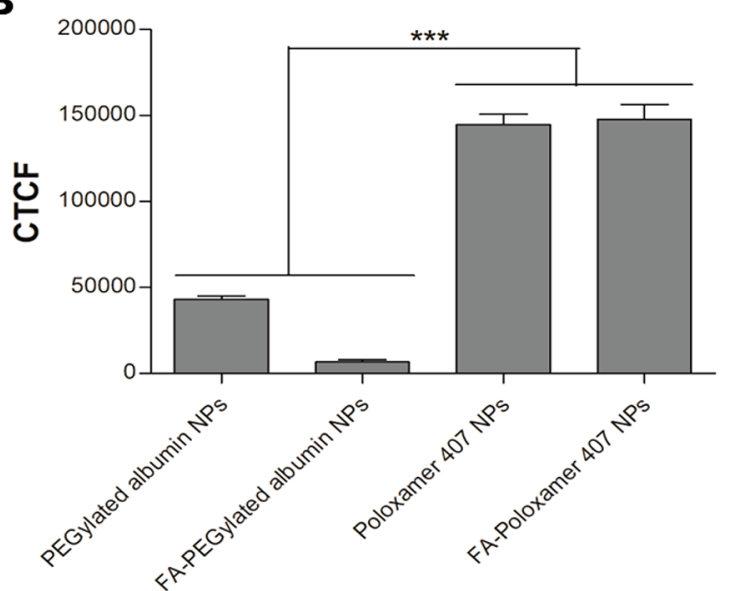

Figure 5. (A) Confocal images of nanoparticle internalization in KB cell line, after $2 \mathrm{~h}$ of incubation. Blue: Hoechst 34580 staining of cell nucleus. Red: nanoparticles loaded with DiI fluorescent probe. (B) CTCF of the cells incubated with the different types of nanoparticles (NPs). Fluorescence intensity was analyzed relatively to DiI.

conjugate was produced by coupling of FA to BSA via carbodiimide linkage between the carboxylic acid groups of FA and the 30-35 lysines containing primary amines that can react with the amine reactive intermediate compound, as previously described by our research group. ${ }^{14}$ Scheme $1 \mathrm{~B}$ shows a schematic illustration of the preparation of FA-Poloxamer 407 nanoparticles. The physicochemical characterization of nanoparticles containing FA showed that the values of size, PDI, and zeta-potential are very similar to that of nontargeted nanoparticles (Table 1). The quantification of FA at the nanoparticle surface, performed by RIDASCREENFAST Folic Acid test kit, revealed a higher FA concentration on Poloxamer 407-based nanoparticles than on the PEGylated albumin nanoparticles. Despite the use of the same initial concentration of FA on both formulations, Poloxamer 407-based nanoparticles presented more FA molecules at their surface. One possible explanation is that FA was linked to hydroxyl groups of PEG hydrophilic chains of Poloxamer 407, which naturally remains more exposed to the nanoparticle surface.

NTA results corroborate the data obtained by DLS analysis (Table 1). NTA allowed us to quantify the nanoparticle concentration on each sample, and similar concentrations of particles per $\mathrm{mL}$ were measured for both PEGylated albuminbased nanoparticles and Poloxamer 407-based nanoparticles.

A key requirement for the application of nanoparticles as drug delivery systems is related to their cytotoxic behavior. This evaluation, using immortalized human normal skin fibroblasts, demonstrated that Poloxamer 407-based nanoparticles (containing or not FA) did not induce loss of cell viability even after $72 \mathrm{~h}$ of contact with different concentrations of nanoparticles (Figure 4), similar to the results described for PEGylated albumin-based nanoparticles. ${ }^{13}$ 
A
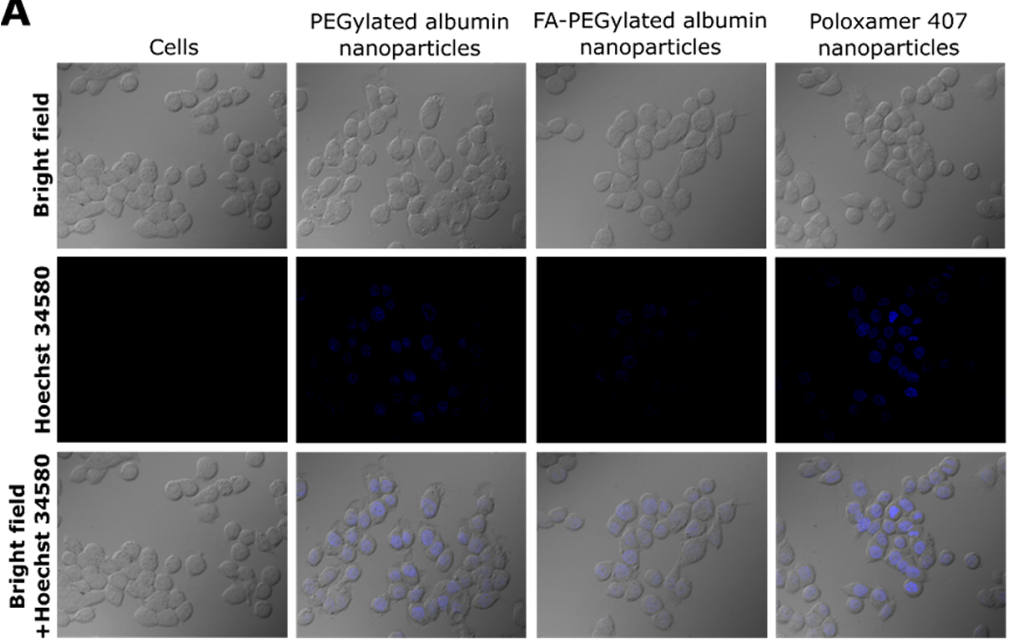

FA-Poloxamer 407 nanoparticles

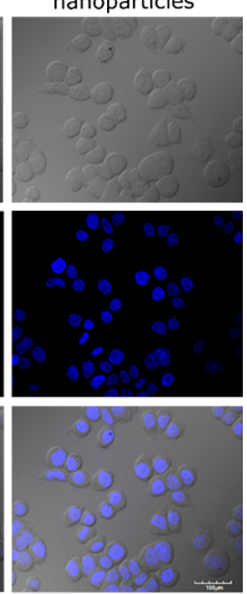

B

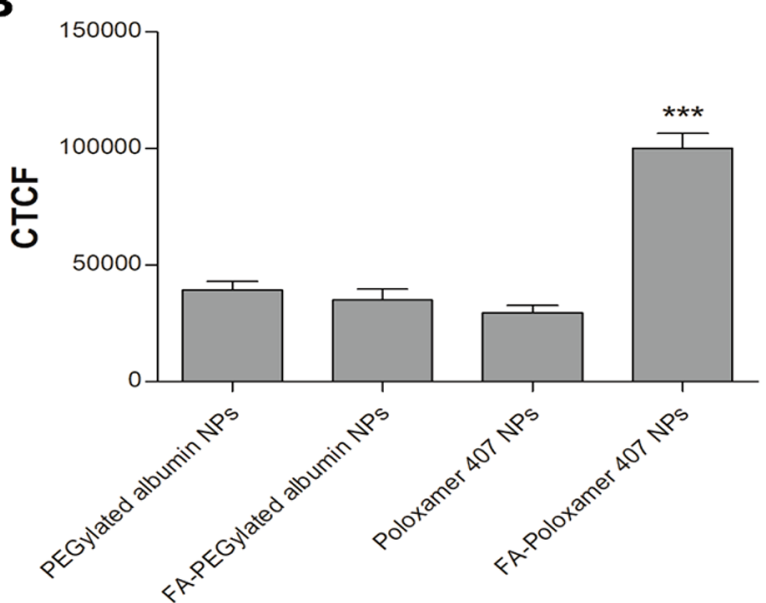

Figure 6. (A) Confocal images of fluorescent nanoparticle internalization and disruption in KB cells, after 2 h of incubation. Blue: Hoechst 34580 staining of cell nucleus. (B) CTCF of the cells incubated with the different types of nanoparticles (NPs). Fluorescence intensity was analyzed relatively to Hoechst 34580 .

\section{Evaluation of Cellular Uptake and Nanoparticle} Disruption. Cellular uptake of nanoparticles loaded with DiI was evaluated by confocal laser scanning microscopy. All samples were prepared using the same solution of vegetable oil containing $4 \mathrm{mg} / \mathrm{mL}$ of DiI. After passage through a gel filtration chromatography column we observed the absence of free dye (loading efficiency $\approx 100 \%$ ), which ensures that all nanoparticles present a similar amount of DiI. The nanoparticles incubated in cell culture medium demonstrated high stability, maintaining their size, PDI, and zeta-potential. Moreover, no free dye was detectable after $2 \mathrm{~h}$ of incubation in cell culture medium. KB cells incubated with Poloxamer 407based nanoparticles exhibited more red fluorescence, evidencing that these nanoparticles are more efficiently internalized by cells than PEGylated albumin-based nanoparticles (Figure 5). The lipid-like amphiphilic nature of Poloxamers allows their effective incorporation into cellular membranes. ${ }^{22}$ Moreover, it has been described that, due to the interaction with plasma membrane, Poloxamers accelerate phospholipid's flip-flop rate and decrease membrane microviscosity, promoting cellular uptake of various small molecules and biomacromolecules. ${ }^{23-25}$ Thus, the well described ability of Poloxamers to bind with cell membrane can be responsible for the efficient internalization of Poloxamer 407-based nanoparticles observed. The presence of albumin on previously reported PEGylated albumin-based nanoparticles can obstruct the interaction of Poloxamer molecules with the cellular membrane, reducing nanoparticles' internalization into cancer cells. Protein molecules may hinder this Poloxamer's ability by decreasing accessibility to the phospholipidic cell membrane.

The release and distribution of the drug into the cells is not usually addressed, only the direct visualization of labeled nanoparticles inside the cells being assessed. In a previous study, our research group effectively verified that Hoechst 34580 encapsulated in nanoparticles can be used as a model to evaluate the in vitro drug release. ${ }^{26}$ This approach described a correlation between the intensity of dye fluorescence and the disruption of the nanoparticles and consequent drug delivery in cell cytoplasm. In this way, Hoechst 34580 was encapsulated in PEGylated albumin-based nanoparticles and Poloxamer 407based nanoparticles and then the nanoparticle disruption was evaluated. The same solution of vegetable oil containing $1 \mathrm{mg} /$ $\mathrm{mL}$ of Hoechst 34580 was used for the nanoparticle preparation. After passage of the nanoparticles through a gel filtration chromatography column, we observed the absence of free dye (loading efficiency $\approx 100 \%$ ), which guarantees that all samples present the same concentration of Hoechst 34580 . This dye is normally used for staining the nuclei of cells because 
its fluorescence is enhanced upon binding to double-stranded DNA. Thus, the internalization and disruption of the nanoparticles were evaluated in terms of nucleus staining. Figure 6 shows that $\mathrm{KB}$ cells incubated with FA-Poloxamer 407 nanoparticles showed stronger blue fluorescence than cells incubated with nontargeted Poloxamer 407-based nanoparticles and with PEGylated albumin-based nanoparticles. These results indicate that FA-Poloxamer 407 nanoparticles have ability to targeted release of the encapsulated compounds, suggesting an efficient interaction between the FA at the surface of nanoparticles and the FR at the cellular surface, promoting FR-mediated endocytosis.

Poloxamer 407-based nanoparticles, compared with PEGylated albumin-based nanoparticles, have lower composition complexity and higher available surface FA concentration. Previous studies demonstrated that a higher concentration of FA does not induce significant differences in nanoparticle internalization. ${ }^{14}$ In this way, we may speculate that the differential cellular uptake and intracellular disruption of nanoparticles is due to the lower composition complexity (absence of albumin) of Poloxamer 407-based nanoparticles, which can present more easily the FA molecules to FR. Compared with the previously developed nanoparticles, Poloxamer 407-based nanoparticles, when internalized, can be more easily degraded by the cell machinery. The presence of albumin in nanoparticle composition would be an additional barrier to achieve the nanoparticle disruption.

\section{CONCLUSION}

In summary, we successfully produced a FA-Poloxamer 407 conjugate by an esterification reaction. Poloxamer 407-based nanoparticles with suitable characteristics for intravenous therapeutic application were developed. Poloxamer 407-based nanoparticles were more efficiently internalized in vitro by cancer cells than PEGylated albumin-based nanoparticles. This result is explained by the well-known capacity of Poloxamers to interact with the cell membrane, potentiating the application of these Poloxamer 407-based nanoparticles as drug delivery systems. Although these new nanoparticles potentiate an efficient interaction with the cell membrane, presenting a higher internalization, our data revealed that only the Poloxamer 407-based nanoparticles containing FA at the surface present an efficient disruption inside the cancer cells. Therefore, the absence of albumin in formulations results in a more effective cellular uptake and nanoparticle disruption, inducing a specific release inside cancer cells.

The developed FA-Poloxamer 407 nanoparticles present suitable characteristics for application as drug delivery systems and are demonstrated to be more effective for application in cancer therapy than the previously developed PEGylated albumin-based nanoparticles. Their production involves lower costs, due to the removal of albumin, resulting in more efficient nanoparticles to interact with MDR cancer cells.

\section{ASSOCIATED CONTENT}

\section{S Supporting Information}

The Supporting Information is available free of charge on the ACS Publications website at DOI: 10.1021/acs.molpharmaceut.7b00893.

${ }^{1} \mathrm{H}$ NMR spectra of Poloxamer 407, FA, and FAPoloxamer 407 conjugate in DMSO- $d_{6}$ (PDF)

\section{AUTHOR INFORMATION}

\section{Corresponding Author}

*E-mail: artur@deb.uminho.pt.

ORCID

Diana Soares da Costa: 0000-0002-1870-6904

Artur Cavaco-Paulo: 0000-0001-7204-2064

Notes

The authors declare no competing financial interest.

\section{ACKNOWLEDGMENTS}

This study was supported by the Portuguese Foundation for Science and Technology (FCT) under the scope of the strategic funding of UID/BIO/04469/2013 unit and COMPETE 2020 (POCI-01-0145-FEDER-006684) and BioTecNorte operation (NORTE-01-0145-FEDER-000004) funded by European Regional Development Fund under the scope of Norte2020-Programa Operacional Regional do Norte. This work has received funding from the European Union Horizon 2020 research and innovation program under Grant Agreement NMP-06-2015-683356 FOLSMART. Diana Soares da Costa thanks Portuguese Foundation for Science and Technology (FCT) for funding her scholarship SFRH/BPD/85790/2012.

\section{REFERENCES}

(1) Batrakova, E. V.; Kabanov, A. V. Pluronic block copolymers: Evolution of drug delivery concept from inert nanocarriers to biological response modifiers. J. Controlled Release 2008, 130 (2), 98-106.

(2) Basak, R.; Bandyopadhyay, R. Encapsulation of Hydrophobic Drugs in Pluronic F127 Micelles: Effects of Drug Hydrophobicity, Solution Temperature, and pH. Langmuir 2013, 29 (13), 4350-4356.

(3) Kabanov, A. V.; Batrakova, E. V.; Alakhov, V. Y. Pluronic ${ }^{\circledR}$ block copolymers as novel polymer therapeutics for drug and gene delivery. J. Controlled Release 2002, 82 (2-3), 189-212.

(4) Adhikari, U.; Goliaei, A.; Tsereteli, L.; Berkowitz, M. L. Properties of Poloxamer Molecules and Poloxamer Micelles Dissolved in Water and Next to Lipid Bilayers: Results from Computer Simulations. J. Phys. Chem. B 2016, 120 (26), 5823-5830.

(5) Kabanov, A. V.; Batrakova, E. V.; Alakhov, V. Y. Pluronic ${ }^{\circledR}$ block copolymers for overcoming drug resistance in cancer. Adv. Drug Delivery Rev. 2002, 54 (5), 759-779.

(6) Chen, Y.; Sha, X.; Zhang, W.; Zhong, W.; Fan, Z.; Ren, Q.; Chen, L.; Fang, $X$. Pluronic mixed micelles overcoming methotrexate multidrug resistance: in vitro and in vivo evaluation. Int. J. Nanomed. 2013, 8, 1463-1476.

(7) Hong, W.; Chen, D.; Zhang, X.; Zeng, J.; Hu, H.; Zhao, X.; Qiao, M. Reversing multidrug resistance by intracellular delivery of Pluronic ${ }^{\circledR}$ P85 unimers. Biomaterials 2013, 34 (37), 9602-9614.

(8) Raval, A.; Pillai, S. A.; Bahadur, A.; Bahadur, P. Systematic characterization of Pluronic ${ }^{\circledR}$ micelles and their application for solubilization and in vitro release of some hydrophobic anticancer drugs. J. Mol. Liq. 2017, 230, 473-481.

(9) Pitto-Barry, A.; Barry, N. P. E. Pluronic block-copolymers in medicine: from chemical and biological versatility to rationalisation and clinical advances. Polym. Chem. 2014, 5 (10), 3291-3297.

(10) Li, Y.-Y.; Li, L.; Dong, H.-Q.; Cai, X.-J.; Ren, T.-B. Pluronic F127 nanomicelles engineered with nuclear localized functionality for targeted drug delivery. Mater. Sci. Eng., C 2013, 33 (5), 2698-2707.

(11) Chen, Y.; Zhang, W.; Huang, Y.; Gao, F.; Sha, X.; Fang, X. Pluronic-based functional polymeric mixed micelles for co-delivery of doxorubicin and paclitaxel to multidrug resistant tumor. Int. J. Pharm. 2015, 488 (1-2), 44-58.

(12) Akash, M. S. H.; Rehman, K. Recent progress in biomedical applications of Pluronic (PF127): Pharmaceutical perspectives. J. Controlled Release 2015, 209, 120-138. 
(13) Loureiro, A.; Nogueira, E.; Azoia, N. G.; Sárria, M. P.; Abreu, A. S.; Shimanovich, U.; Rollett, A.; Härmark, J.; Hebert, H.; Guebitz, G.; Bernardes, G. J. L.; Preto, A.; Gomes, A. C.; Cavaco-Paulo, A. Size controlled protein nanoemulsions for active targeting of folate receptor positive cells. Colloids Surf., B 2015, 135, 90-98.

(14) Loureiro, A.; Abreu, A. S.; Sarria, M. P.; Figueiredo, M. C. O.; Saraiva, L. M.; Bernardes, G. J. L.; Gomes, A. C.; Cavaco-Paulo, A. Functionalized protein nanoemulsions by incorporation of chemically modified BSA. RSC Adv. 2015, 5 (7), 4976-4983.

(15) Lowry, O. H.; Rosebrough, N. J.; Farr, A. L.; Randall, R. J. Protein measurement with the folin phenol reagent. J. Biol. Chem. 1951, 193 (1), 265-275.

(16) Kim, G. J.; Nie, S. Targeted cancer nanotherapy. Mater. Today 2005, 8 (8, Supplement), 28-33.

(17) Loureiro, A.; Azoia, N. G.; Gomes, A. C.; Cavaco-Paulo, A. Albumin-Based Nanodevices as Drug Carriers. Curr. Pharm. Des. 2016, 22 (10), 1371-1390.

(18) Zhang, W.; Shi, Y.; Chen, Y.; Ye, J.; Sha, X.; Fang, X. Multifunctional Pluronic P123/F127 mixed polymeric micelles loaded with paclitaxel for the treatment of multidrug resistant tumors. Biomaterials 2011, 32 (11), 2894-2906.

(19) Lin, J.-J.; Chen, J.-S.; Huang, S.-J.; Ko, J.-H.; Wang, Y.-M.; Chen, T.-L.; Wang, L.-F. Folic acid-Pluronic F127 magnetic nanoparticle clusters for combined targeting, diagnosis, and therapy applications. Biomaterials 2009, 30 (28), 5114-5124.

(20) Eisele, K.; Gropeanu, R.; Musante, A.; Glasser, G.; Li, C.; Muellen, K.; Weil, T. Tailored Albumin-based Copolymers for Receptor-Mediated Delivery of Perylenediimide Guest Molecules. Macromol. Rapid Commun. 2010, 31 (17), 1501-1508.

(21) Hao, H.; Ma, Q.; Huang, C.; He, F.; Yao, P. Preparation, characterization, and in vivo evaluation of doxorubicin loaded BSA nanoparticles with folic acid modified dextran surface. Int. J. Pharm. 2013, 444 (1-2), 77-84.

(22) Guo, D.-D.; Moon, H.-S.; Arote, R.; Seo, J.-H.; Quan, J.-S.; Choi, Y.-J.; Cho, C.-S. Enhanced anticancer effect of conjugated linoleic acid by conjugation with Pluronic F127 on MCF-7 breast cancer cells. Cancer Lett. 2007, 254 (2), 244-254.

(23) Alakhova, D. Y.; Kabanov, A. V. Pluronics and MDR Reversal: An Update. Mol. Pharmaceutics 2014, 11 (8), 2566-2578.

(24) Peetla, C.; Stine, A.; Labhasetwar, V. Biophysical Interactions with Model Lipid Membranes: Applications in Drug Discovery and Drug Delivery. Mol. Pharmaceutics 2009, 6 (5), 1264-1276.

(25) Lai, T. C.; Kataoka, K.; Kwon, G. S. Pluronic-based cationic block copolymer for forming pDNA polyplexes with enhanced cellular uptake and improved transfection efficiency. Biomaterials 2011, 32 (20), 4594-4603.

(26) Nogueira, E.; Cruz, C. F.; Loureiro, A.; Nogueira, P.; Freitas, J.; Moreira, A.; Carmo, A. M.; Gomes, A. C.; Preto, A.; Cavaco-Paulo, A. Assessment of liposome disruption to quantify drug delivery in vitro. Biochim. Biophys. Acta, Biomembr. 2016, 1858 (2), 163-167. 